\title{
Dynamical Systems, the Three-Body Problem and Space Mission Design
}

\author{
Wang Sang Koon, Martin W. Lo, Jerrold E. Marsden, Shane D. Ross \\ Control and Dynamical Systems, Caltech and JPL, Pasadena, California, USA \\ International Conference on Differential Equations, Berlin, 1999 \\ Edited by B. Fiedler, K. Gröger and J. Sprekels, \\ World Scientific, 2000, 1167-1181.
}

\begin{abstract}
This paper concerns heteroclinic connections and resonance transitions in the planar circular restricted 3-body problem, with applications to the dynamics of comets and asteroids and the design of space missions such as the Genesis Discovery Mission and low energy Earth to Moon transfers. The existence of a heteroclinic connection between pairs of equal energy periodic orbits around two of the libration points is shown numerically. This is applied to resonance transition and the construction of orbits with prescribed itineraries. Invariant manifold structures are relevant for transport between the interior and exterior Hill's regions, and other resonant phenomena throughout the solar system.
\end{abstract}

\section{Introduction.}

Resonant Transition in Comet Orbits. Some Jupiter comets such as Oterma and Gehrels 3 make a rapid transition from heliocentric orbits outside the orbit of Jupiter to orbits inside that of Jupiter and vice versa. During this transition, the comet may be captured temporarily by Jupiter for several orbits. The interior orbit is typically close to the $3: 2$ resonance while the exterior orbit is near the 2:3 resonance. During the transition, the orbit passes close to the libration points $L_{1}$ and $L_{2}$, two of the five equilibrium points (in a rotating frame) for the planar circular restricted 3-body problem (PCR3BP) for the Sun-Jupiter system. The equilibrium points $L_{1}$ and $L_{2}$ are the ones closest to Jupiter, lying on either side of Jupiter along the Sun-Jupiter line, with $L_{1}$ being between Jupiter and the sun.

The Relevance of Invariant Manifolds. Belbruno and B. Marsden [1997] considered the comet transitions using the "fuzzy boundary" concept. Lo and Ross [1997] used the PCR3BP as the underlying model and related it to invariant manifolds, noticing that the orbits of Oterma and Gehrels 3 (in the Sun-Jupiter rotating frame) closely follow the invariant manifolds of $L_{1}$ and $L_{2}$. We develop this viewpoint along with another key ingredient, a heteroclinic connection between periodic orbits around $L_{1}$ and $L_{2}$ with the same Jacobi constant (a multiple of the energy for the PCR3BP) and the dynamical consequences of such an orbit. Invariant manifold structures associated with $L_{1}$ and $L_{2}$ periodic orbits and the heteroclinic connections assist in the understanding of transport throughout the solar system. We have drawn upon work of the Barcelona group on the PCR3BP, in particular, Llibre, Martinez and Simó [1985] as well as works of Moser, Conley and McGehee. Specific citations are given later.

Heteroclinic Connections. A numerical demonstration is given of a heteroclinic connection between pairs of equal energy periodic orbits, one around $L_{1}$, the other around $L_{2}$. This heteroclinic connection augments the previously known homoclinic orbits associated with the $L_{1}$ and $L_{2}$ periodic orbits. Linking these heteroclinic connections and homoclinic orbits leads to dynamical chains which form the backbone for temporary capture and rapid resonance transition of Jupiter comets. See Figure 1.1. 


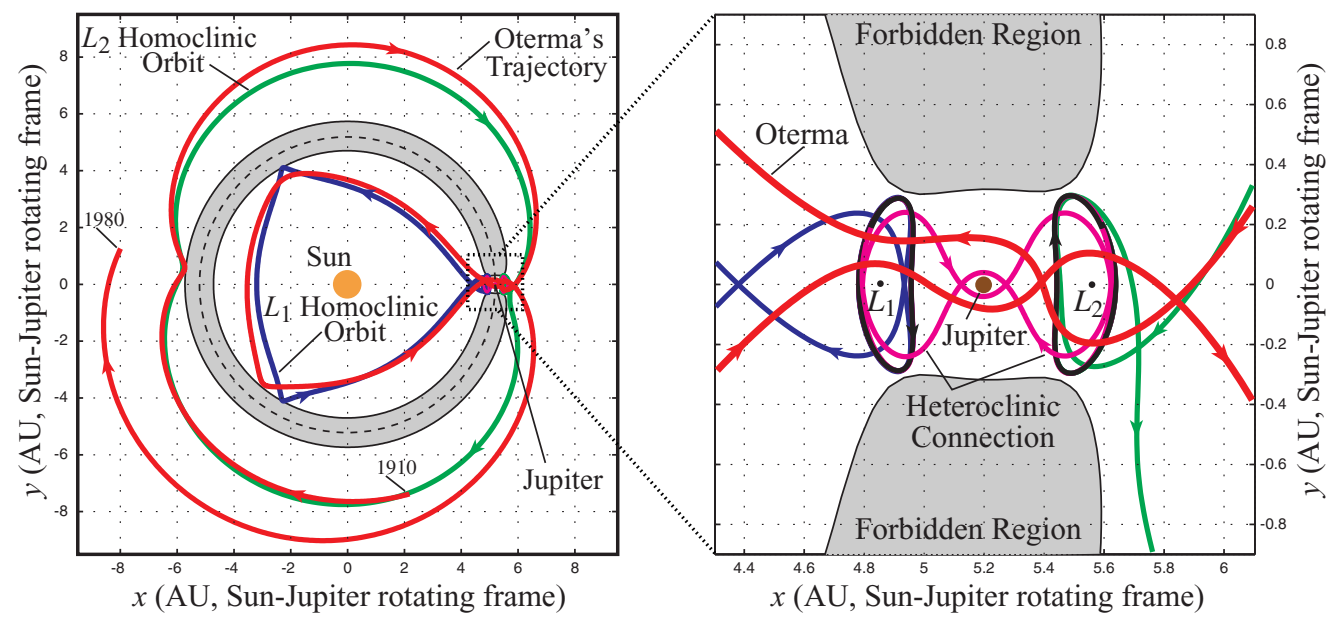

Figure 1.1: The homoclinic-heteroclinic chain corresponding to the Jupiter comet Oterma. Shown are the periodic orbits about $L_{1}$ and $L_{2}$, their homoclinic orbits and the heteroclinic connection between them. The orbit of Oterma (AD 1910-1980) is shown overlaying the chain. Distances are in Astronomical Units (AU).

Existence and Construction of Transition Orbits. We have proved the existence of a large class of interesting orbits near a chain which a comet can follow in its rapid transition between the inside and outside of Jupiter's orbit via a Jupiter encounter. The collection of these orbits is called a dynamical channel. We also use this term when collections of such chains for separate 3-body systems, roughly speaking, overlap and are put end to end. We can label orbits near a chain with an itinerary giving their past and future whereabouts, making their classification and manipulation possible. We prove the existence of orbits with prescribed itineraries, and develop a systematic procedure for their numerical construction.

Applications to Space Mission Design. The systematic procedures developed here can be used to design spacecraft orbits which explore a large region of space in the vicinity of the Earth (and near Earth's orbit) using low-fuel controls. Behavior related to the dynamical channels has already been observed in the trajectory for NASA's Genesis Discovery Mission, which exhibits near-heteroclinic motion between $L_{1}$ and $L_{2}$ in the Sun-Earth system (Lo et al. [1998]). A better understanding of the homoclinic-heteroclinic structures allows one to construct and control spacecraft trajectories with desired characteristics. Specifically, these techniques can be used to construct a "Petit Grand Tour" of the moons of Jupiter. We can design an orbit which follows a prescribed itinerary in its visit to the many moons (e.g., one orbit around Ganymede, four around Europa, etc.). See Figure 1.2.

Earth to Moon Transfer with Ballistic Capture. We apply similar techniques used to generate the "Petit Grand Tour" to produce a lunar capture mission which uses less fuel than a Hohmann transfer. We approximately decouple the Sun-Earth-Moon-Spacecraft 4-body problem into two 3-body problems. Using the invariant manifold theory of the Lagrange points of the 3-body systems, we construct low energy transfer trajectories from the Earth to a ballistic capture at the Moon.

Optimal Control and Halo Orbit Mission. We address the computation of the required trajectory correction maneuvers (TCM) for a halo orbit space mission (like Genesis) to compensate for the launch velocity errors introduced by inaccuracies of the launch vehicle. By combining dynamical systems theory with optimal control techniques, we produce a portrait of the complex landscape of the trajectory design space. This approach enables parametric studies not available to mission designers a few years ago, such as how the magnitude of the errors and the timing of the first TCM affect the correction $\Delta V$. 




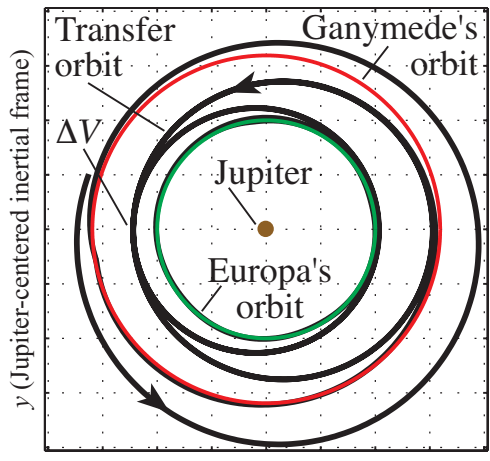

$x$ (Jupiter-centered inertial frame)

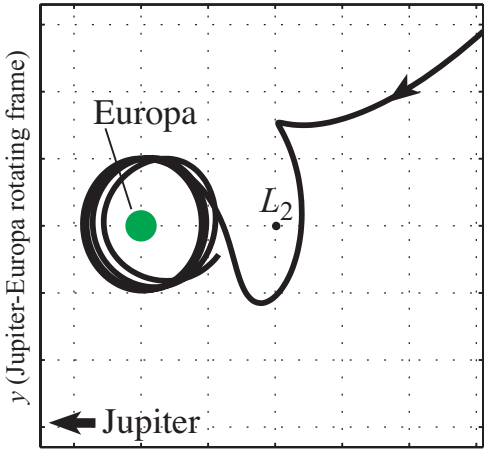

$x$ (Jupiter-Europa rotating frame)

Figure 1.2: The "Petit Grand Tour" space mission concept for the Jovian moons. In our example, we show an orbit coming into the Jupiter system and (a) performing one loop around Ganymede (shown in the Jupiter-Ganymede rotating frame), (b) transferring from Ganymede to Europa using a single impulsive maneuver (shown in the Jupiter-centered inertial frame), and (c) getting captured by Europa (shown in the Jupiter-Europa rotating frame).

\section{A Few Key Features of the Three Body Problem.}

The Planar Circular Restricted Three-Body Problem. The comets of interest are mostly heliocentric, but their perturbations are dominated by Jupiter's gravitation. Moreover, their motion is nearly in Jupiter's orbital plane, and Jupiter's small eccentricity (0.0483) plays little role during the fast resonance transition (less than or equal to one Jupiter period in duration). The PCR3BP is therefore an adequate starting model for illuminating the essence of the resonance transition process.

Equations of Motion. The two main bodies, such as the Sun and Jupiter, have masses $m_{S}=1-\mu$ and $m_{J}=\mu$, so the total mass that is normalized to one. (See Figure 2.1(a)). These bodies rotate in the plane counterclockwise about their common center of mass and with the angular velocity normalized to one. The third body, the comet or the spacecraft, has small mass that does not affect the primary motion, and is free to move in the plane. Choose a rotating coordinate system so that the origin is at the center of mass and the Sun $(S)$ and Jupiter $(J)$ are fixed on the $x$-axis at $(-\mu, 0)$ and $(1-\mu, 0)$ respectively. Let $(x, y)$ be the position of the comet in the plane (so these are the position coordinates relative to the positions of the Sun and Jupiter, not relative to an inertial frame).

The equations of motion of the comet are (see, eg, Szebehely [1967])

$$
\ddot{x}-2 \dot{y}=\Omega_{x}, \quad \ddot{y}+2 \dot{x}=\Omega_{y}, \quad \text { where } \quad \Omega=\frac{x^{2}+y^{2}}{2}+\frac{1-\mu}{r_{1}}+\frac{\mu}{r_{2}}+\frac{\mu(1-\mu)}{2},
$$

$\Omega_{x}, \Omega_{y}$ are the partial derivatives of $\Omega$ and $r_{1}$ and $r_{2}$ are the distances of the comet from the main bodies. A first integral, the Jacobi integral is $C(x, y, \dot{x}, \dot{y})=-\left(\dot{x}^{2}+\dot{y}^{2}\right)+2 \Omega(x, y)=-2 E(x, y, \dot{x}, \dot{y})$, where $E$ is the energy. For a Hamiltonian approach, see Abraham and Marsden [1978] or Meyer and Hall [1992].

Equilibrium Points and Hill's Regions The system (2.1) has five equilibrium points, 3 collinear ones on the $x$-axis, called $L_{1}, L_{2}, L_{3}$ and two equilateral points called $L_{4}, L_{5}$. The value of the Jacobi integral at the point $L_{i}$ will be denoted by $C_{i}$. The level surfaces of the Jacobi constant, which are also energy surfaces, are invariant 3-dimensional manifolds. Let $\mathcal{M}$ be that energy surface, i.e., $\mathcal{M}(\mu, C)=$ $\{(x, y, \dot{x}, \dot{y}) \mid C(x, y, \dot{x}, \dot{y})=$ constant $\}$. The projection of this surface onto position space is called a Hill's region, $M(\mu, C)=\{(x, y) \mid \Omega(x, y) \geq C / 2\}$. The boundary of $M(\mu, C)$ is the zero velocity curve. The comet can move only within this region in the $(x, y)$-plane. We study orbits whose Jacobi constant is just below that of $L_{2}$, that is, $C<C_{2}$. For this case, the Hill's region contains a "neck" about $L_{1}$ and $L_{2}$, as shown in Figure 2.1(b). Thus, orbits with a Jacobi constant just below that of $L_{2}$ are energetically 
permitted to make a transit through the neck region from the interior region (inside Jupiter's orbit) to the exterior region (outside Jupiter's orbit) passing through the Jupiter region.

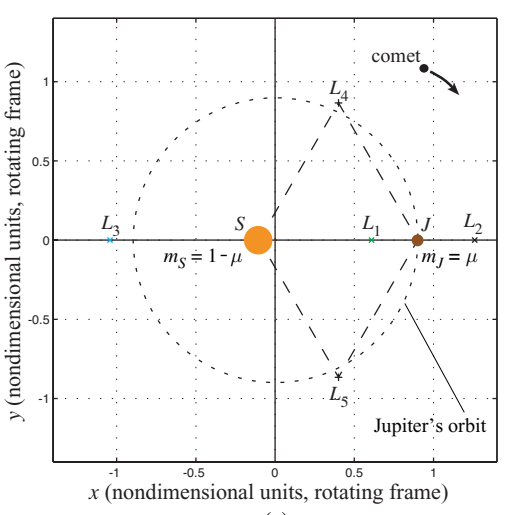

(a)

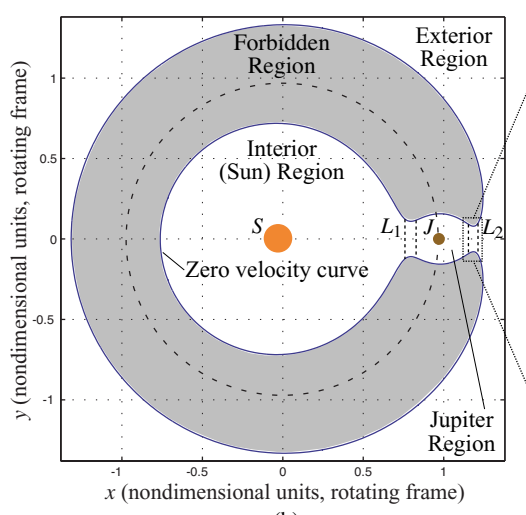

(b)

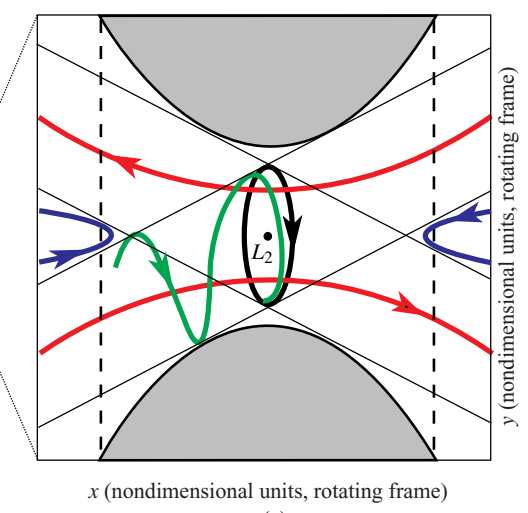

(c)

Figure 2.1: (a) Equilibrium points of the PCR3BP as viewed in the rotating frame. (b) Hill's region (schematic, the region in white), which contains a "neck" about $L_{1}$ and $L_{2}$. (c) The flow in the region near $L_{2}$, showing a periodic orbit, a typical asymptotic orbit, two transit orbits and two non-transit orbits. A similar figure holds for the region around $L_{1}$.

The Flow near the Lagrange Points $L_{1}$ and $L_{2}$. Having fixed on an appropriate energy level surface, a major result of Conley [1963, 1968] on the flow near the equilibrium points $L_{1}$ and $L_{2}$ shows that in each equilibrium region around $L_{1}$ or $L_{2}$, there are four types of orbits (see Figures 2.1(c)): the periodic Liapunov orbit, the asymptotic orbits that wind onto this periodic orbit, the transit orbits that the comet uses to make a transit from one region to the other and the non-transit orbits where the comet comes out of one region and passes near the critical point only to fall back into the same region.

\section{Heteroclinic Connections and Resonance Transition.}

We give a numerical demonstration of a heteroclinic connection between a pair of equal energy periodic orbits, one around $L_{1}$ and one around $L_{2}$. This heteroclinic connection augments the homoclinic orbits associated with the $L_{1}$ and $L_{2}$ periodic orbits, which were previously known. By linking these heteroclinic connections and homoclinic orbits, we get dynamical chains which form the backbone for temporary capture and rapid resonance transition of Jupiter comets. See Figure 1.1.

The Existence of Heteroclinic Connections between Periodic Orbits. To find a heteroclinic connection between the periodic orbits mentioned in the previous paragraph, we find an intersection of their respective invariant manifolds in the $\mathcal{J}$ region (Jupiter region of phase space) within a suitably chosen Poincaré section. For instance, to generate a heteroclinic orbit which goes from an $L_{1}$ periodic orbit (as $t \rightarrow-\infty$ ) to an $L_{2}$ periodic orbit (as $t \rightarrow+\infty$ ), we proceed as follows. Let the branch of the unstable manifold of the $L_{1}$ periodic orbit which enters the $\mathcal{J}$ region be denoted $W_{L_{1} \text {,p.o. }}^{u, \mathcal{J}}$. On the same energy surface there is an $L_{2}$ periodic orbit, whose stable manifold in the $\mathcal{J}$ region we shall similarly denote $W_{L_{2}, \text { p.o. }}^{s, \mathcal{J}}$. The projections of the two-dimensional manifold tubes onto position space are shown in Figure 3.1(a). To find intersections between these two tubes, we cut the flow by the plane $x=1-\mu$, as in Figure 3.1(b). The $q$-th intersection of $W_{L_{1}, \text { p.o. }}^{u, \mathcal{J}}$ with the plane $x=1-\mu$ will be labeled $\Gamma_{L_{1}, q}^{u, \mathcal{J}}$. Similarly, we will call $\Gamma_{L_{2}, p}^{s, \mathcal{J}}$ the $p$-th intersection of $W_{L_{2}, \text { p.o. }}^{s, \mathcal{J}}$ with $x=1-\mu$. Numerical computations show that (for small $p$ and $q$ ), the $L_{1}$ periodic orbit unstable manifold $W_{L_{1}, \text { p.o. }}^{u, \mathcal{J}}$ and the $L_{2}$ periodic orbit stable manifold $W_{L_{2}, \text { p.o. }}^{s, \mathcal{J}}$ are closed curves that intersect transversally. A point in the plane $x=1-\mu$ belonging 


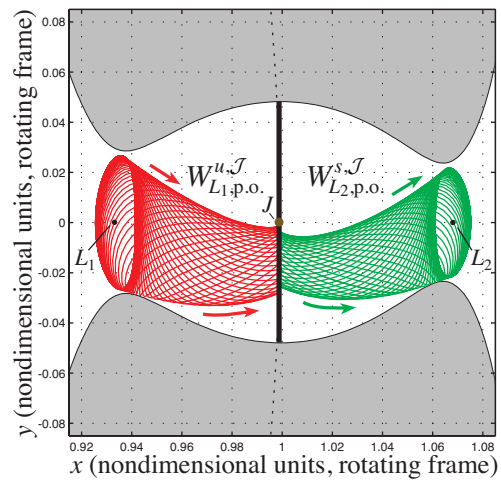

(a)

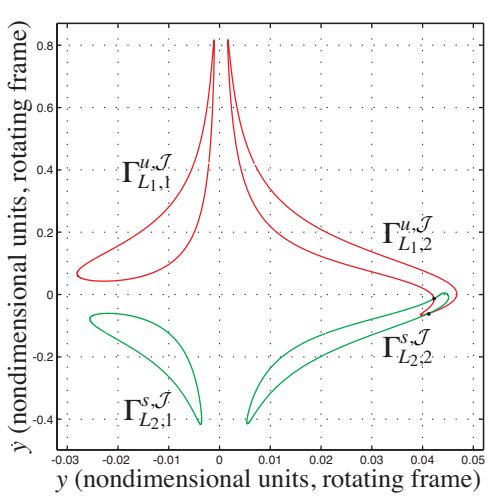

(b)

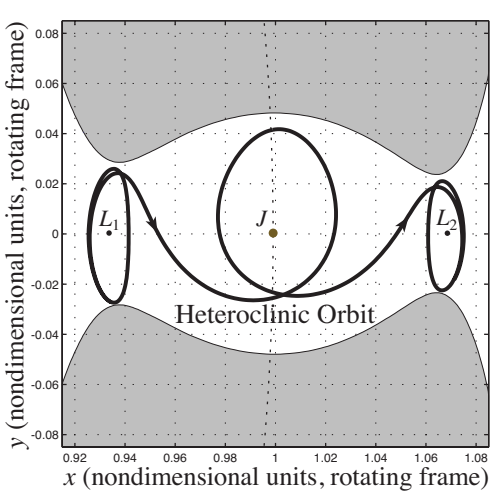

(c)

Figure 3.1: (a) The projection of invariant manifolds $W_{L_{1}, \text { p.o. }}^{u, \mathcal{J}}$ and $W_{L_{2}, \text { p.o. }}^{s, \mathcal{J}}$ in the region $J$ of the position space. (b) The first two Poincaré cuts of the invariant manifolds with the plane $x=1-\mu$. (c) The existence of a transversal $(2,2)$-heteroclinic orbit in the $J$ region.

to $\Gamma_{L_{1}, q}^{u, \mathcal{J}} \cap \Gamma_{L_{2}, p}^{s, \mathcal{J}}$ is called a $(q, p)$-heteroclinic point because such a point corresponds to a heteroclinic orbit going from the $L_{1}$ Liapunov orbit to the $L_{2}$ Liapunov orbit.

Figure 3.1(b) shows the curves $\Gamma_{L_{1}, q}^{u, \mathcal{J}}$ for $q=1,2$ and $\Gamma_{L_{2}, p}^{s, \mathcal{J}}$ for $p=1,2$. Notice that $\Gamma_{L_{1}, 2}^{u, \mathcal{J}}$ and $\Gamma_{L_{2}, 2}^{s, \mathcal{J}}$ intersect in two points (the dots in Figure 3.1(b)). Thus, the minimum $q$ and $p$ for a heteroclinic point to appear for this particular value of $C$ is $q=2$ and $p=2$. The $(2,2)$-heteroclinic points can each be forward and backward integrated to produce heteroclinic trajectories going from the $L_{1}$ Liapunov orbit to the $L_{2}$ Liapunov orbit. We show one of the heteroclinic orbits in Figure 3.1(c).

Chains of Homoclinic Orbits and Heteroclinic Cycles. Equations (2.1) have the symmetry $s:(x, y, \dot{x}, \dot{y}, t) \rightarrow(x,-y,-\dot{x}, \dot{y},-t)$, so if we know the unstable manifold of the periodic orbit, the corresponding stable manifold is obtained using symmetry. This observation can be used to find the reverse trajectory, going from the $L_{2}$ periodic orbit to the $L_{1}$ periodic orbit. It would be the mirror image (about the $x$-axis) of the trajectory in Figure 3.1(c), with the direction arrows reversed. These two heteroclinic connections together form a symmetric heteroclinic cycle. Similarly, we can also use a combination of the same analytical and numerical techniques to show the existence of transversal homoclinic orbits associated to the $L_{1}$ and $L_{2}$ periodic orbits in both the interior and exterior regions.

Combining homoclinic and heteroclinic orbits of the same energy, generates a homoclinic-heteroclinic chain, which connect asymptotically the $L_{1}$ and $L_{2}$ periodic orbits to each other. As will be seen, these chains imply a complicated dynamics connecting the interior, exterior, and Jupiter regions. In the next section, we shall state a slightly simplified version of a theorem in Koon et al. [1999a]. This theorem shows the existence of a large class of interesting orbits near such a chain which a comet can follow in its rapid transition between the interior and the exterior regions passing through the Jupiter region.

Resonance Transtion of Comet Oterma. We choose the Sun-Jupiter system $(\mu=0.0009537)$, and a Jacobi constant value similar to that of Oterma during its Jupiter encounters $(C=3.03)$. Using the above methods, we get an interior region orbit homoclinic to the $L_{1}$ periodic orbit, an exterior region orbit homoclinic to the $L_{2}$ periodic orbit, and a heteroclinic cycle connecting the $L_{1}$ and $L_{2}$ periodic orbits. The union of these orbits is a homoclinic-heteroclinic chain. See Figure 1.1. Note that the interior homoclinic orbit is in 3:2 resonance with Jupiter and the exterior homoclinic orbit is in 2:3 resonance with Jupiter. Observe how closely the orbit of Oterma shadows this chain. Therefore, it is reasonable for us to conclude that the dynamical channels near this chain provide the orbits for such a transition. 


\section{Existence and Numerical Construction of Transitional Orbits.}

To state the main theorem of Koon et al. [1999a] precisely, we recall some basic ideas of global bifurcation theory. For more details, consult Moser [1973], Llibre, Martinez and Simó [1985] and Wiggins [1990].

The idea of reducing the study of the global orbit structure to the study of an associated discrete map is due to Poincaré, who utilized the method in his studies of the restricted 3-body problem. Koon et al. [1999a] use the chain of two homoclinic orbits and one symmetric heteroclinic cycle (generated above) to construct a suitable Poincaré map. Our choice of Poincaré map allows us to study the complex global orbit structure near the chain. We find an invariant set for this map near some transversal homoclinic and heteroclinic points along the chain where "Smale horseshoe"-like dynamics exist. We then use symbolic dynamics to characterize the chaotic motion in a neighborhood of the chain as it transitions intermittently through the interior, Jupiter and exterior regions.

Construction of Poincaré Map with Domain $U$. We construct (Figure 4.1) a Poincaré map $P$ transversal to the flow whose domain $U$ consists of four different squares $U_{i}, i=1,2,3,4$, located in different regions of phase space in the neighborhood of the chain. Squares $U_{1}$ and $U_{4}$ are contained in

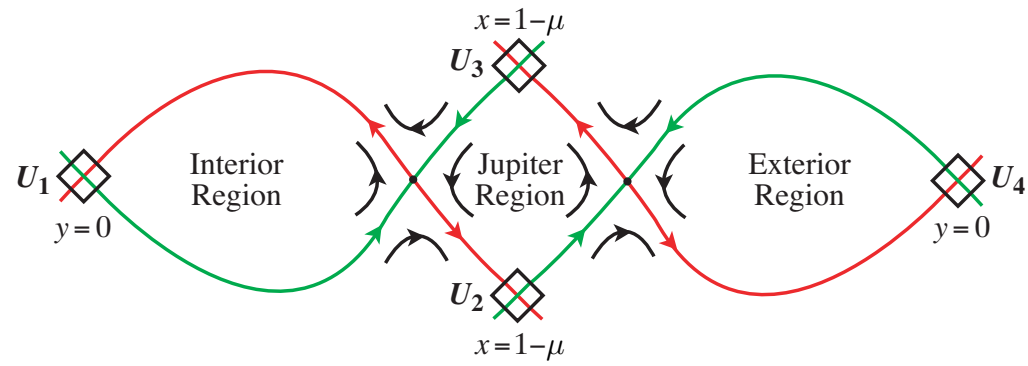

Figure 4.1: The construction of a suitable Poincaré map.

the surface $y=0$ and each centers around a transversal homoclinic point in the interior and the exterior region, respectively. Squares $U_{2}$ and $U_{3}$ are contained in the surface $x=1-\mu(y<0$ and $y>0$, respectively) and center around transversal heteroclinic points in the Jupiter region which are symmetric with respect to each other. Clearly, for any orbit which passes through a point $q$ in one of the squares and whose images and pre-images $\left(P^{n}(q), n=0, \pm 1, \pm 2, \ldots\right)$ all remain in the domain $U$, the whereabouts of $P^{n}(q)$ (as $n$ increases or decreases) can provide some of the essential information about the history of the particular orbit. We record this history with a bi-infinite sequence. This technique of studying only the set of points that forever remain in the domain $U$ (the invariant set $\Lambda=\cap_{n=-\infty}^{\infty} P^{n}(U)$ ) provides us with all the periodic solutions as well as the recurrent solutions in the neighborhood of the chain.

Application of Symbolic Dynamics. We construct a set of bi-infinite sequences with two families of symbols. The first family is a subshift of finite type with four symbols $\left\{u_{1}, u_{2}, u_{3}, u_{4}\right\}$, used to track an orbit with respect to the four squares $U_{1}, U_{2}, U_{3}, U_{4}$. The symbol $u_{i}$ is recorded every time the $U_{i}$ square is pierced by the orbit. Subshift here means that among the set of all bi-infinite sequences of four symbols, (i.e., $\left(\ldots, u_{i_{-1}} ; u_{i_{0}}, u_{i_{1}}, u_{i_{2}}, \ldots\right)$ where $i_{j}$ ranges from 1 to 4 ), certain sequences where the adjacent entries in the sequence violate certain relations are not allowed. For example, from $U_{1}$, the (forward) flow cannot get to $U_{4}$ without passing through other squares. Hence, in the bi-infinite sequence, the symbol $u_{1}$ cannot be followed by $u_{4}$. The relations can be defined by the transition matrix: $(A)_{k l}=1$ if the ordered pair of symbols $u_{k}, u_{l}$ may appear as adjacent entries in the symbolic sequence, and $(A)_{k l}=0$ if the ordered pair of symbols $u_{k}, u_{l}$ may not appear as adjacent entries. For example, since $u_{1}$ cannot be followed by $u_{4}$, we have $(A)_{14}=0$. The second family is a full shift of infinite type with symbols of positive integers greater than a fixed integer $r_{\min }$. This set of bi-infinite sequences of positive integers is used to keep track of the number of integer revolutions that the projection of an orbit winds around either $L_{1}$ or $L_{2}$ when the orbit enters the equilibrium regions $\mathcal{R}_{1}$ or $\mathcal{R}_{2}$, respectively. 
Main Theorem on Global Orbit Structure. We state a simplified version of the main theorem of Koon et al. [1999a], which provides a symbolic dynamics description of the global orbit structure of the PCR3BP near a chain $\mathcal{C}$ of homoclinic orbits and a symmetric heteroclinic cycle. Suppose $\mathcal{C}$ is made up of a symmetric $\left(q_{2}, p_{2}\right)$-heteroclinic cycle in the Jupiter region together with two homoclinic orbits, one of which is a $\left(q_{1}, p_{1}\right)$ orbit in the interior region and the other is a $\left(q_{3}, p_{3}\right)$ orbit in the exterior region.

Theorem 4.1. Consider an element $(u, r) \in \bar{\Sigma}$ with $r_{j} \geq r_{\min }$ for all $j$. Then there are initial conditions, unique in a neighborhood of the given chain of two homoclinic orbits and one symmetric heteroclinic cycle, such that the following statements are true.

For an element of type $\alpha=\left(\ldots,\left(u_{i_{-1}}, r_{-1}\right) ;\left(u_{i_{0}}, r_{0}\right),\left(u_{i_{1}}, r_{1}\right),\left(u_{i_{2}}, r_{2}\right), \ldots\right)$, the orbit corresponding to such conditions starts at $U_{i_{0}}$ and goes to $U_{i_{1}}$ if $(A)_{i_{0} i_{1}}=1$. This orbit passes through either the equilibrium region $\mathcal{R}_{1}$ or $\mathcal{R}_{2}$ depending on whether the initial index $i_{0}$ is 1,3 or 2,4 . If $i_{0}=1,3$, the projection of the orbit winds around $L_{1}$ for $r_{0}$ revolutions inside the region $\mathcal{R}_{1}$ before leaving for $U_{i_{1}}$. Otherwise, it winds around $L_{2}$ for $r_{0}$ revolution before leaving for $U_{i_{1}}$. After that, the same process begins with $\left(u_{i_{1}}, r_{1}\right)$ in place of $\left(u_{i_{0}}, r_{0}\right)$ and $\left(u_{i_{2}}, r_{2}\right)$ in place of $\left(u_{i_{1}}, r_{1}\right)$, etc. For negative time a similar behavior is described for $\left(u_{i_{-1}}, r_{-1}\right),\left(u_{i_{0}}, r_{0}\right)$, etc.

For this orbit, the number of revolutions that the comet winds around Jupiter or the Sun (in the interior or exterior region) is a constant which depends on the region and the given chain of homoclinic orbits and heteroclinic cycle. For the Jupiter region, the number is $\left(q_{2}+p_{2}-1\right) / 2$. For the interior and exterior regions, the number is $q_{1}+p_{1}-1$ and $q_{3}+p_{3}-1$ respectively.

Numerical Construction of Orbits with Prescribed Itineraries. We have studied the complex orbit structure in the neighborhood of a chain. What we have found is an invariant set of orbits, to each of which we can attach an itinerary (e.g., $(\ldots, X, J, S, J, \ldots)$ in the informal notation) describing the future and past history of the orbit for all time. Here, the symbols $\{S, J, X\}$ denote the location of the orbit in the interior (Sun), Jupiter, or exterior regions, respectively. Furthermore, Theorem 4.1 shows us that all permissible itineraries exist in the neighborhood of a chain.

While the invariant set is a useful theoretical construct for the classification of the dynamics, its infinite nature does not directly give usable trajectories. However, guided by the proof, computational and numerical methods can be brought to bear which iteratively approximate the invariant set and allow us to develop a systematic procedure to numerically construct orbits with finite prescribed itineraries.

The construction of the invariant set uses successive iterations of the Poincare map $P$. Finite areas of finite central block itineraries evolved under successive application of the map $P$ into a "cloud of points," the invariant set $\Lambda$. If we truncate the construction of the invariant set at some finite number of iterations of $P$, we find regions of phase space with a finite itinerary. The itinerary of an orbit in this region is robust in the sense that all nearby orbits in phase space have the same finite itinerary. Thus, by truncating our construction of the invariant set $\Lambda$ at some finite number of applications of $P$, we generate a set of robust orbits with different finite itineraries. The sets of orbits with different itineraries are easily visualizable on our chosen Poincaré section as areas in which all the orbits have the same finite itinerary. We will also no longer be limited to a small neighborhood of a chain, but can obtain more global results.

Example Itinerary: $(J, X ; J, S, J)$. We illustrate the numerical construction of orbits with prescribed finite (but arbitrarily large) itineraries. As our example, chosen for simplicity of exposition, we construct an orbit with the central block $(J, X ; J, S, J)$. To link the present numerical construction with the earlier theoretical framework, we adopt the following convention. The $U_{1}$ and $U_{4}$ (Poincaré) sections will be the planes $(y=0, x<0)$ in the interior region, and $(y=0, x<-1)$ in the exterior region, respectively. The $U_{2}$ and $U_{3}$ sections will be the planes $(x=1-\mu, y<0)$ and $(x=1-\mu, y>0)$ in the Jupiter region, respectively. A key observation here is a result of McGehee [1969] which has shown that the invariant manifold tubes separate two types of motion. See Figures 4.2(a) and 4.2(b). The orbits inside the tube transit from one region to another; those outside the tubes bounce back to their original region.

Since the upper curve in Figure 4.2(b) is the Poincaré cut of the stable manifold of the periodic orbit around $L_{1}$ in the $U_{3}$ plane, a point inside that curve is an orbit that goes from the Jupiter region to the 

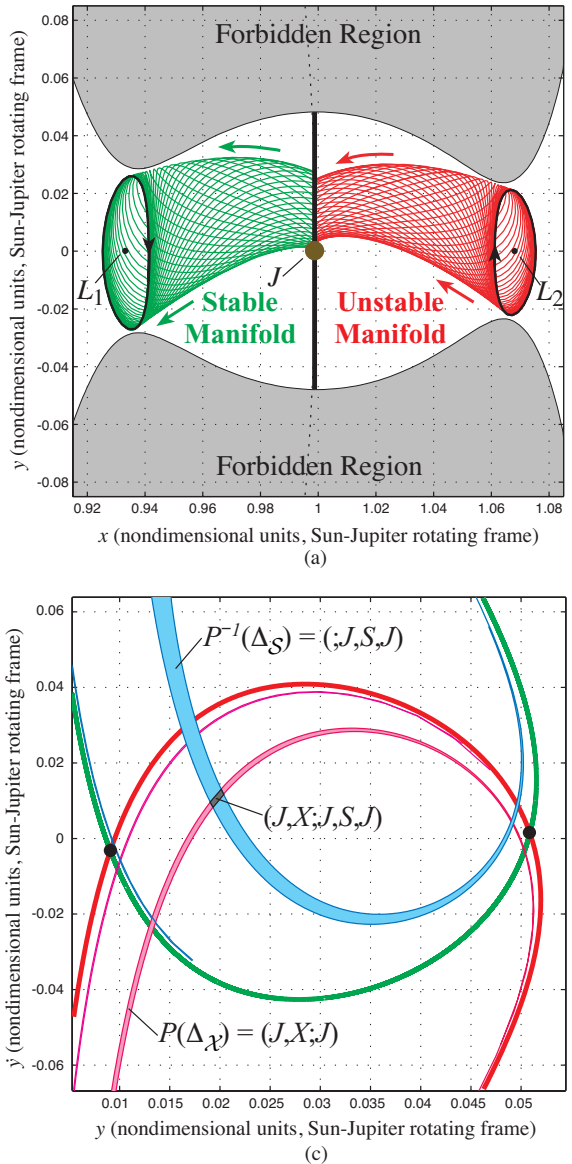

(c)

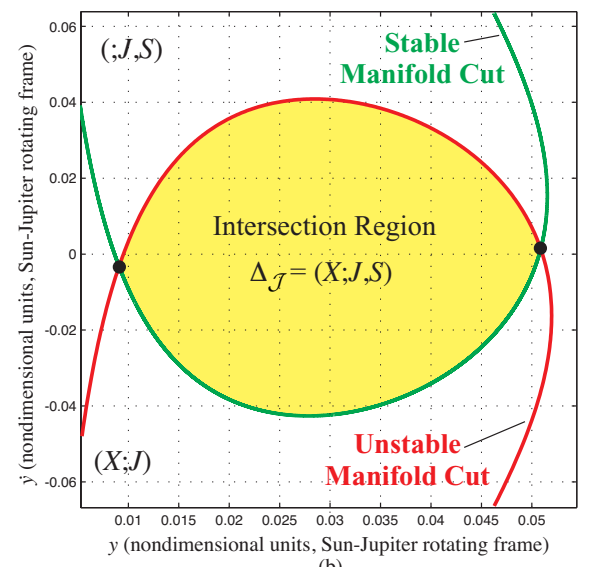

(b)

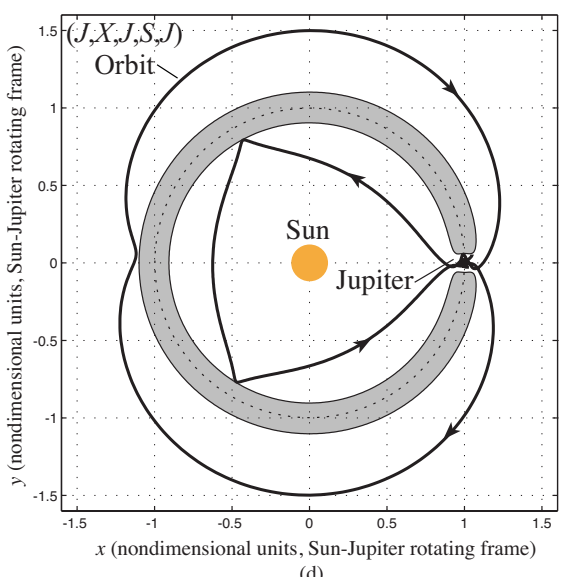

(d)

Figure 4.2: (a) The projection of invariant manifolds $W_{L_{1}, \text { p.o. }}^{s, \mathcal{J}}$ and $W_{L_{2}, \text { p.o. }}^{u, \mathcal{J}}$ in the region $J$ of the position space. (b) A close-up of the intersection region between the Poincaré cuts of the invariant manifolds on the $U_{3}$ section $(x=1-\mu, y>0)$. (c) Intersection between image of $\Delta_{X}$ and pre-image of $\Delta_{S}$ labeled $(J, X ; J, S, J)$. (d) Example orbit passing through $(J, X ; J, S, J)$ region of (c).

interior region, so this region can be described by the label $(; J, S)$. Similarly, a point inside the lower curve of Figure 4.2(b) came from the exterior region into the Jupiter region, and so has the label $(X ; J)$. A point inside the intersection $\Delta_{J}$ of both curves is an $(X ; J, S)$ orbit, so it makes a transition from the exterior region to the interior region, passing through the Jupiter region. Similarly, by choosing Poincaré sections in the interior and the exterior region, i.e., in the $U_{1}$ and $U_{4}$ plane, we find the intersection region $\Delta_{S}$ consisting of $(J ; S, J)$ orbits, and $\Delta_{X}$, which consists of $(J ; X, J)$ orbits.

Flowing the intersection $\Delta_{X}$ forward to the Jupiter region, it stretches into the strips in Figure 4.2(c). These strips are the image of $\Delta_{X}$ (i.e., $\left.P\left(\Delta_{X}\right)\right)$ under the Poincaré map $P$, and thus get the label $(J, X ; J)$. Similarly, flowing the intersection $\Delta_{S}$ backward to the Jupiter region, it stretches into the strips $P^{-1}\left(\Delta_{S}\right)$ in Figure $4.2(\mathrm{c})$, and thus have the label $(; J, S, J)$. The intersection of these two types of strips (i.e., $\left.\Delta_{J} \cap P\left(\Delta_{X}\right) \cap P^{-1}\left(\Delta_{S}\right)\right)$ consist of the desired $(J, X ; J, S, J)$ orbits. If we take any point inside these intersections and integrate it forward and backward, we find the desired orbits. See Figure $4.2(\mathrm{~d})$. 


\section{Earth to Moon Transfer with Ballistic Capture}

Shoot the Moon. The techniques developed thus far, especially the construction of orbits with prescribed itineraries, have been used to produce the "Petit Grand Tour" of Jovian moons mentioned above (see Figure 1.2). Similar techniques also produce a low-fuel transfer to the Moon, like the 1991 Hiten mission which effected a ballistic capture at the Moon with less propulsion than a standard Hohmann transfer (Belbruno and Miller [1993]). We refer to this mission concept as "Shoot the Moon."

Our approach to effect an Earth to Moon transfer with a ballistic ("free") capture at the Moon builds on the theoretical understanding developed in Koon et al. [1999a] as well as the following three key ideas: (1) treat the Sun-Earth-Moon-Spacecraft 4-body problem as two coupled circular restricted 3body problems, Sun-Earth-SC and Earth-Moon-SC systems, (2) use the stable and unstable manifolds of periodic orbits about the Sun-Earth Lagrange points to provide a low energy transfer from Earth to the stable manifolds of periodic orbits around the Earth-Moon Lagrange points, (3) use the stable manifolds of the periodic orbits around the Earth-Moon Lagrange points to provide a ballistic capture about the Moon. The construction is done mainly in the Sun-Earth rotating frame using a Poincaré section which help to glue the Sun-Earth libration point trajectory with the lunar ballistic capture trajectory.

We start with the PCR3BP model to compute the invariant manifolds and use them to construct the Sun-Earth libration point trajectory and the lunar ballistic capture trajectory. The final trajectory starting from the Earth and ending in lunar capture is integrated in the model of 4-body bi-circular problem where both the Moon and the Earth are assumed to move in circular orbits about the Earth and the Sun respectively in the ecliptic, and the spacecraft is an infinitesimal mass point. Details of the "Shoot the Moon" concept appears in Koon et al. [2000].

Earth to Moon Transfer Mechanism. To effect the Earth to Moon transfer, we compute Sun-Earth invariant manifolds of an $L_{2}$ periodic orbit, which leave the vicinity of the Earth and enter the region of the Earth-Moon manifolds. We seek an orbit that launches from a $200 \mathrm{~km}$ altitude Earth parking orbit and is drawn into a ballistic capture by the Moon. This involves the stable/unstable manifold symmetry and the behavior of orbits near the outside of the stable/unstable manifold tubes (Koon et al. [1999a]).

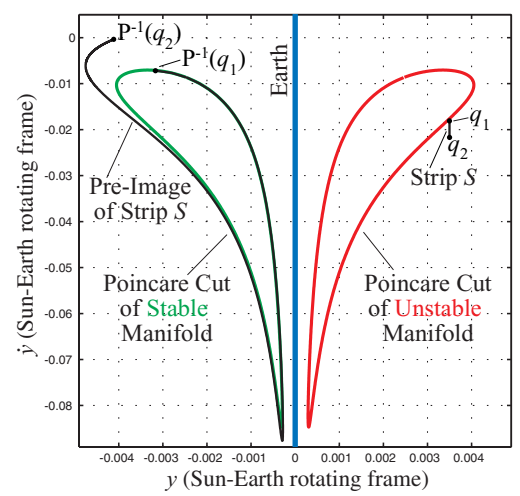

(a)

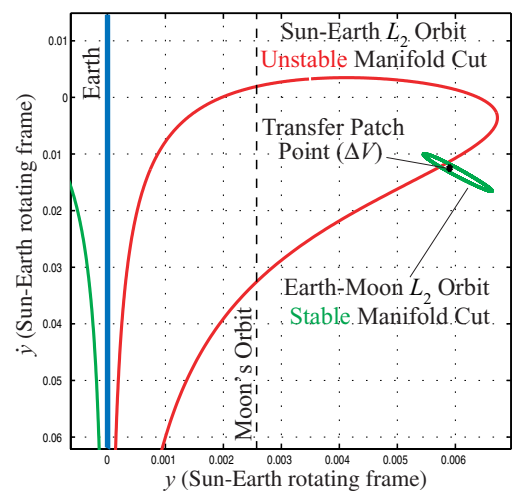

(b)



(c)

Figure 5.1: (a) Behavior of orbits near and outside the stable and unstable manifolds of the Sun-Earth $L_{2}$ orbit in the Earth region. (b) Intersection between Sun-Earth $L_{2}$ orbit unstable manifold and Earth-Moon $L_{2}$ orbit stable manifold (small oval). (c) The Earth to Moon transfer with ballistic capture by the Moon.

We select Sun-Earth $L_{2}$ periodic orbits within a range of energies, and compute their stable and unstable manifolds in the Earth region. For a certain energy, consider a strip $S$ (line with endpoints $q_{1}$ and $q_{2}$ ) near the outside of the unstable manifold cut (right loop in Figure 5.1(a)) on a Poincaré section passing through the Earth at $x=1-\mu$. The points in this strip all have the same position, but they differ in $y$-velocity. Using the general theory described in Koon et al. [1999a], it is known that 
the points in strip $S$ wind around the equilibrium point $L_{2}$ for a different number of revolutions when integrated backward. Since the orbits in the strip are outside of the unstable manifold tube, they will fall back toward the Poincare section. In other words, the pre-image of the strip $P^{-1}(S)$ wind around the stable manifold (left loop). Using this observation, note that for fixed position, the slightest change in $\dot{y}$ (effected by a small $\Delta V$, propulsive change in velocity) can move the pre-image of a point near the unstable manifold cut to anywhere near the Poincarś cut of the stable manifold. Thus, for a point in the Poincaré section near and outside the unstable manifold cut, we can use a small $\Delta V$ to take us back to a $200 \mathrm{~km}$ altitude Earth parking orbit near the outside of the stable manifold. We will therefore restrict our Sun-Earth-SC 3-body energy to the range where the parking orbit position is near the stable manifold.

Lunar Ballistic Capture Trajectory. Next we obtain a trajectory approaching the Moon. The stable and unstable manifolds of the Sun-Earth $L_{2}$ orbit draw the spacecraft away from Earth and into large revolutions about the Earth outside of the Moon's orbit. This suggests using the stable manifolds of the Earth-Moon $L_{2}$ orbit to draw the spacecraft into the Moon's vicinity for ballistic capture. We patch these two trajectories where the Sun-Earth and Earth-Moon $L_{2}$ orbit manifolds intersect.

Each trajectory within the Poincaré cut of the stable manifold (small oval in Figure 5.1(b)) is transported into the Moon region, where it will perform several circuits about the Moon in a state of temporary capture. Among the points outside of but close to the Sun-Earth $L_{2}$ orbit unstable manifold cut, there is a trajectory which departs from a $200 \mathrm{~km}$ altitude Earth parking orbit. If we now also require this point to fall within the Earth-Moon $L_{2}$ orbit stable manifold, it is guaranteed to approach the Moon. Since the energies of the Sun-Earth and Earth-Moon manifolds differ slightly, the intersections produced in this manner require a small $\Delta V$. By selecting appropriate energies properly in both systems, this small $\Delta V$ may be eliminated, meaning a completely free transfer to lunar capture is possible!

We use the above procedure to find initial guesses in the coupled 3-body model which we adjust finely to obtain a true 4-body bi-circular model trajectory. Figure 5.1(c) is the final end-to-end trajectory in inertial coordinates. A $\Delta V$ of $34 \mathrm{~m} / \mathrm{s}$ is required at the location marked. Using the trajectory from the bi-circular model, an end-to-end trajectory can be computed in the JPL ephemeris model using JPL's LTool (Libration Point Mission Design Tool) currently under development. This computation is the subject of a future paper.

\section{Optimal Control for Halo Orbit Missions}

This section describes some results of Serban et al. [2000] in the larger context of the present paper. Genesis is a solar wind sample return mission. It is one of NASA's first robotic sample return missions, scheduled for launch in January 2001 to a 3-dimensional halo orbit in the vicinity of the Sun-Earth $L_{1}$ Lagrange point. Once there, the spacecraft will remain for two years to collect solar wind samples before returning them to the Earth for study. The Genesis trajectory (see Figure 6.1(a)) was designed using dynamical systems theory. The three year mission, from launch all the way to Earth return, requires only a single small deterministic maneuver (less than $6 \mathrm{~m} / \mathrm{s}$ ) when injecting onto the halo orbit.

The Trajectory Correction Maneuver (TCM) Problem. The most important error in the launch of Genesis is the launch velocity error. The one sigma expected error is $7 \mathrm{~m} / \mathrm{s}$ for a boost of nearly 3200 $\mathrm{m} / \mathrm{s}$ from a circular $200 \mathrm{~km}$ altitude Earth orbit. Such an error is large because halo orbit missions are extremely sensitive to launch errors. Typical planetary launches can correct launch vehicle errors 7 to 14 days after the launch. This correction maneuver is called TCM1, being the first TCM of any mission. In contrast, for orbits such as the Genesis transfer trajectory, the correction maneuver $\Delta V$ grows sharply in inverse proportion to the time from launch. For a large launch vehicle error, which is possible in Genesis' case, the TCM1 can quickly grow beyond the capability of the spacecraft's propulsion system.

The Genesis spacecraft, built in the spirit of NASA's new low cost mission approach, is very basic. This makes the performance of an early TCM1 difficult and risky. It is desirable to delay TCM1 as long as possible, even at the expense of expenditure of the $\Delta V$ budget. In fact, Genesis would prefer TCM1 be 

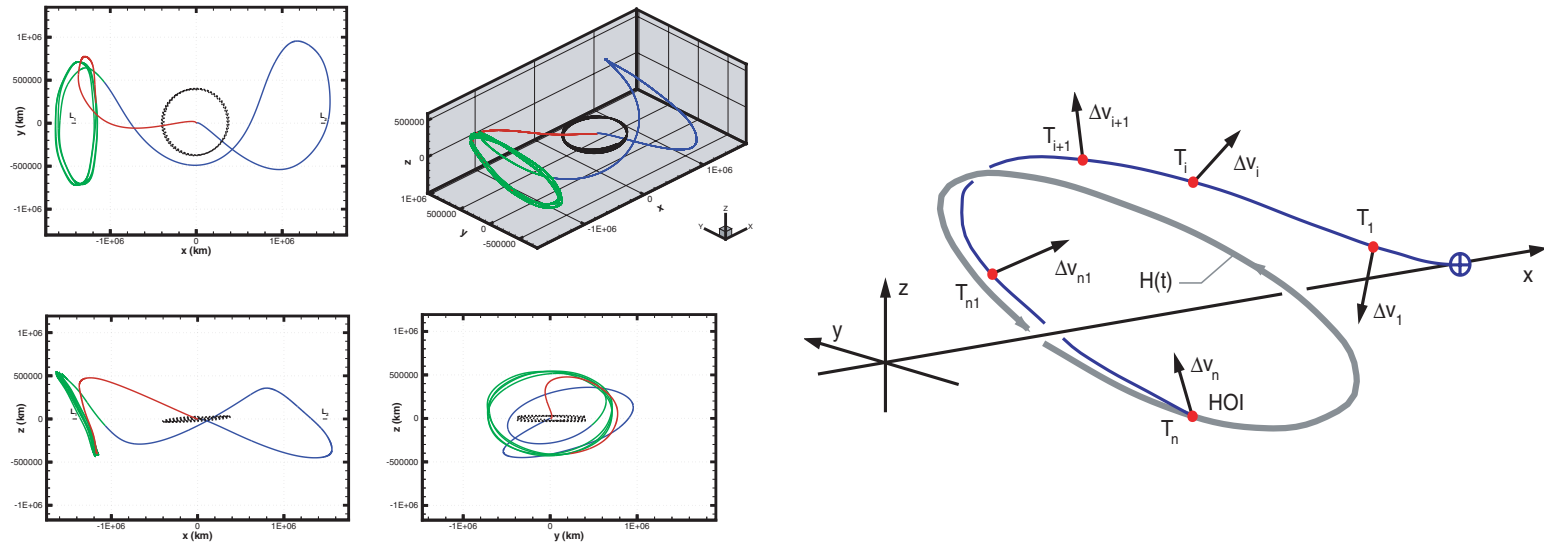

(a)

(b)

Figure 6.1: (a) The Genesis Discovery Mission trajectory. (b) Schematic of optimal control trajectory correction maneuver problem.

performed at 2 to 7 days after launch, or even later. However, beyond launch +24 hours, the correction $\Delta V$ based on traditional linear analysis can become prohibitively high.

The desire to increase the time between launch and TCM1 compels one to use a nonlinear approach, based on combining dynamical systems theory with optimal control techniques. In joint work with Serban and Petzold of the University of California, Santa Barbara, we have explored two similar but slightly different approaches and are able to obtain in both cases an optimal maneuver strategy that fits within the Genesis $\Delta V$ budget of $450 \mathrm{~m} / \mathrm{s}$. (1) HOI technique: we use optimal control techniques to retarget the halo orbit with the original nominal trajectory as the initial guess. (2) MOI technique: we target the stable manifold. Both methods yield good results. In this paper, we will discuss only briefly the HOI technique. For more details, see Serban et al. [2000].

Recasting TCM as Trajectory Planning Problem. We recast the TCM problem as a spacecraft trajectory planning optimization problem. The $\Delta V$ caused by perturbation in the launch velocity and by different delays in TCM1 becomes exactly the sensitivity analysis of the optimal solution. COOPT, the software package we use is an excellent tool in solving this type of problem, both in providing an optimal solution and in studying the sensitivity of different parameters. It is developed by the Computational Science and Engineering Group at UC Santa Barbara (see Users' Guide [1999]).

A halo orbit insertion trajectory design problem can be simply posed as:

Find the maneuver times and sizes to minimize fuel consumption ( $\Delta V$ ) for a trajectory starting near Earth and ending on the specified $L_{1}$ halo orbit at a position and with a velocity consistent with the HOI time.

We assume that the evolution of the spacecraft is described by a set of six ODEs, $\dot{\mathbf{x}}=\mathbf{f}(t, \mathbf{x})$ where $\mathbf{x}=\left[\mathbf{x}^{p} ; \mathbf{x}^{v}\right] \in R^{6}$ contains both positions $\left(\mathbf{x}^{p}\right)$ and velocities $\left(\mathbf{x}^{v}\right)$. In this paper, we use the 3-dimensional CR3BP model; other models will be investigated in future work.

To deal with the discontinuous nature of the control force $(\Delta V)$, the equations of motion are solved simultaneously on each interval between two maneuvers. Let the maneuvers $M_{1}, M_{2}, \ldots, M_{n}$ take place at times $T_{i}, i=1,2, \ldots, n$ and let $\mathbf{x}_{i}(t), t \in\left[T_{i-1}, T_{i}\right]$ be the solution on the interval $\left[T_{i-1}, T_{i}\right]$ (see Figure 6.1(b)). Position continuity constraints are imposed at each maneuver, that is, $\mathbf{x}_{i}^{p}\left(T_{i}\right)=\mathbf{x}_{i+1}^{p}\left(T_{i}\right), i=$ $1,2, \ldots, n-1$. In addition, the final position is forced to lie on the halo orbit, that is, $\mathbf{x}_{n}^{p}\left(T_{n}\right)=\mathbf{x}_{H}^{p}\left(T_{n}\right)$, where the halo orbit is parameterized by the HOI time $T_{n}$. Additional constraints dictate that the first maneuver is delayed by at least a prescribed amount $T C M 1_{\min }$ after launch, that is, $T_{1} \geq T C M 1_{\text {min }}$. 
With a cost function $C\left(\Delta \mathbf{v}_{i}\right)=\sum_{i=1}^{n}\left\|\Delta \mathbf{v}_{i}\right\|$. defined as some measure of the velocity discontinuities $\Delta \mathbf{v}_{i}=\mathbf{x}_{i+1}^{v}\left(T_{i}\right)-\mathbf{x}_{i}^{v}\left(T_{i}\right), i=1,2, \ldots, n-1$, and $\Delta \mathbf{v}_{n}=\mathbf{x}_{H}^{v}\left(T_{n}\right)-\mathbf{x}_{n}^{v}\left(T_{n}\right)$, the optimization problem becomes $\min _{T_{i}, \mathbf{x}_{i}, \Delta \mathbf{v}_{i}} C\left(\Delta \mathbf{v}_{i}\right)$, subject to the constraints.

In many optimal control problems, obtaining an optimal solution is not the only goal. The influence of problem parameters on the optimal solution (the so called sensitivity of the optimal solution) is also needed. In this paper, we are interested in estimating the $(\Delta V)$ caused by perturbations in the launch velocity $\left(\epsilon_{0}^{v}\right)$ and by different delays in the first maneuver (TCM1). In COOPT, we make use of the Sensitivity Theorem to give us the desired sensitivity results.

Halo Orbit Insertion (HOI) Problem. In this problem we directly target the selected halo orbit with the last maneuver taking place at the HOI point. Using the optimization procedure described above, we compute the optimal cost transfer trajectories for various combinations of $T C M 1_{\text {min }}$ (ranging from 1 day to 5 days) and $\epsilon_{0}^{v}$ (ranging from $-7 \mathrm{~m} / \mathrm{s}$ to $+7 \mathrm{~m} / \mathrm{s}$ ).

The optimization procedure was applied for all values of $T C M 1_{\min }$ and $\epsilon_{0}^{v}$ in the ranges of interest. We first investigated the possibility of correcting for errors in the launch velocity using at most two maneuvers $(n=2)$. Except for the cases in which there is no error in the launch velocity (and for which the final optimal transfer trajectories have only one maneuver at HOI), the first correction maneuver is always on the prescribed lower bound $T C M 1_{\text {min }}$. For all cases investigated, halo orbit insertion takes place at most 18.6 days earlier or 28.3 days later than in the nominal case $\left(T_{H O I}=110.2\right.$ days $)$.

Several conclusions can be drawn. The main contribution of dynamical systems theory to the computation of optimal trjaectory correction maneuvers is in the construction of good initial guess trajectories in sensitive regions. Moreover, for all cases that we investigated, the optimal costs are well within the $\Delta V$ budget allocated for trajectory correction maneuvers $(450 \mathrm{~m} / \mathrm{s}$ for the Genesis mission). The cost function is very close to being linear with respect to both $T C M 1_{\min }$ time and launch velocity error. Also, the halo orbit insertion time is always close enough to that of the nominal trajectory as not to affect either the collection of the solar wind or the rest of the mission.

\section{Conclusions and Future Work.}

This paper used dynamical systems techniques to investigate heteroclinic connections and resonance transitions in the PCR3BP. A main result is the discovery of a heteroclinic connection between $L_{1}$ and $L_{2}$ equal energy periodic orbits and the dynamical description of nearby orbits. This augments the known homoclinic orbits associated to the $L_{1}$ and $L_{2}$ Liapunov periodic orbits (McGehee [1969] and Llibre, Martinez and Simó [1985]). By linking these heteroclinic connections with homoclinic orbits on the same energy surface, we find dynamical channels providing a fast transport mechanism between the interior and exterior Hill's regions (not to be confused with Arnold diffusion). This should contribute to the transport mechanisms connecting mean motion resonances, and in particular, those mechanisms linking interior and exterior resonances via the Jupiter capture region. By comparing observations of the orbits of Jupiter comets like Oterma with these dynamical channels, we conclude that the comets are guided by them (Figure 1.1). Moreover, these dynamical channels could be exploited by spacecraft to explore a large region of space around any planet or moon system using low-fuel controls.

Extension to Three Dimensions. We plan to extend our techniques to the 3-dimensional CR3BP by seeking homoclinic and heteroclinic orbits associated with 3-dimensional periodic "halo" and quasiperiodic "quasi-halo" and Lissajous orbits about $L_{1}$ and $L_{2}$. Their union is a set of 3-dimensional homoclinic-heteroclinic chains around which symbolic dynamics could catelog a variety of exotic orbits.

The 3-dimensional chains would provide an initial template for the construction of actual spacecraft trajectories. By presenting a more complete portrait of the phase space geometry near $L_{1}$ and $L_{2}$, the 3-dimensional channels will be of enormous benefit in the design and control of constellations of spacecraft in these regions. The homoclinic-heteroclinic structures suggest natural low-fuel paths for deployment of constellation spacecraft to and from Earth. 
Coupling of Two Three-Body Systems. To obtain a better grasp of the dynamics governing transport between adjacent planets (or moons), we could apply our methodology to the coupled PCR3BP. The coupled PCR3BP considers two nested co-planar 3-body systems, such as for two adjacent giant planets competing for control of the same comet (e.g., Sun-Jupiter-comet and Sun-Saturn-comet). When close to the orbit of one of the planets, the comet's motion is dominated by the corresponding planet's 3-body dynamics. Between the two planets, the comet's motion is mostly heliocentric, but is precariously poised between two competing 3-body dynamics. In this region, heteroclinic orbits connecting periodic orbits of two different 3-body systems may exist, leading to complicated transfer dynamics between two adjacent planets. See Figure 1.2.

This transfer dynamics, which may be realized in actual comet behavior, could be exploited for free transfers of spacecraft between adjacent moons in the Jovian and Saturnian systems (Lo and Ross [1998]). For instance, one could conduct a "Petit Grand Tour" of the Jovian moon system, an example of which is shown in Figure 1.2. By systematically seeking heteroclinic connections between libration point orbits of adjacent moons, one could design trajectories which transfer from the vicinity of one moon to another using little fuel. Furthermore, the "Shoot the Moon" trajectory suggests the use of exotic low-fuel transfers between a planet and its moon (see Figure 5.1(c)).

Merging Optimal Control and Stabilization with Dynamical Systems Theory. The construction of exotic spacecraft orbits using homoclinic-heteroclinic chains requires optimal, fuel minimizing impulsive and continuous propulsion thruster controls to navigate these dynamically sensitive regions of phase space. A deeper understanding of the dynamical structure of the restricted 3-body problem may suggest alternative formulations of the optimizing scheme which are based more on the geometry of the phase space. Algorithms could be developed with the natural dynamics built in, thereby yielding better convergence properties. It would also be interesting to explore additional ways to use optimal control in the presence of mechanics (as in, for example, Koon and Marsden [1997]).

In addition to the optimal control problem of getting to a halo orbit, there are well known techniques for stabilizing the dynamics once one gets there. Some techniques are related to the general theory of stabilizing dynamics near saddle points and homoclinic or heteroclinic orbits, as in Bloch and Marsden [1989]. In addition, it would be of interest to explore the use of other stabilization techniques that make use of the mechanical structure for problems of this sort, as in Bloch, Leonard and Marsden [1997].

Symplectic Integrators. The use of symplectic integrators for the long time integrations of solar system dynamics is well known through the work of Tremaine, Wisdom and others. The area of integration algorithms for mechanical systems continues to develop and be implemented; see for example, Kane, Marsden and Ortiz [1999], and Kane, Marsden, Ortiz and West [1999] and references therein. These techniques are very effective for both conservative mechanical systems as well as systems with forcing, such as controlled systems. It would be of interest to explore these numerical methods in the context of space mission design and other orbital mechanics problems.

Four or More Body Problems. While the PCR3BP model provides an adequate explanation for a class of Jupiter comets whose Jacobi constant is close to (and less than) $C_{2}$ and whose motion is close to the plane of Jupiter's orbit, it fails to explain resonance transition phenomena for high inclination Jupiter comets and comets not dominated solely by Jupiter. For these, other effects such as out-of-plane motion and perturbation by other giant planets, notably Saturn, are quite strong and need to be considered. The study of this second class of comets require the complete storehouse of tools needed in the study of the near-Earth asteroids, regarded by many as the most challenging topic in celestial mechanics.

Since mean motion resonances (mostly with Jupiter) and associated transport mechanisms play the dominant role in solar system material transport, the general theory of Koon et al. [199a] lays a foundation for future studies in this direction. We may need to consider other more complicated models like the full 3-dimensional CR3BP and the coupled PCR3BP as mentioned above. As Lo and Ross [1997] suggested, further exploration of the phase space structure as revealed by the homoclinic-heteroclinic structures and their association with mean motion resonances may provide deeper conceptual insight into the evolution and structure of the asteroid belt (interior to Jupiter) and the Kuiper Belt (exterior to Neptune), plus 
the transport between these two belts and the terrestrial planet region. See Figure 7.1(a).
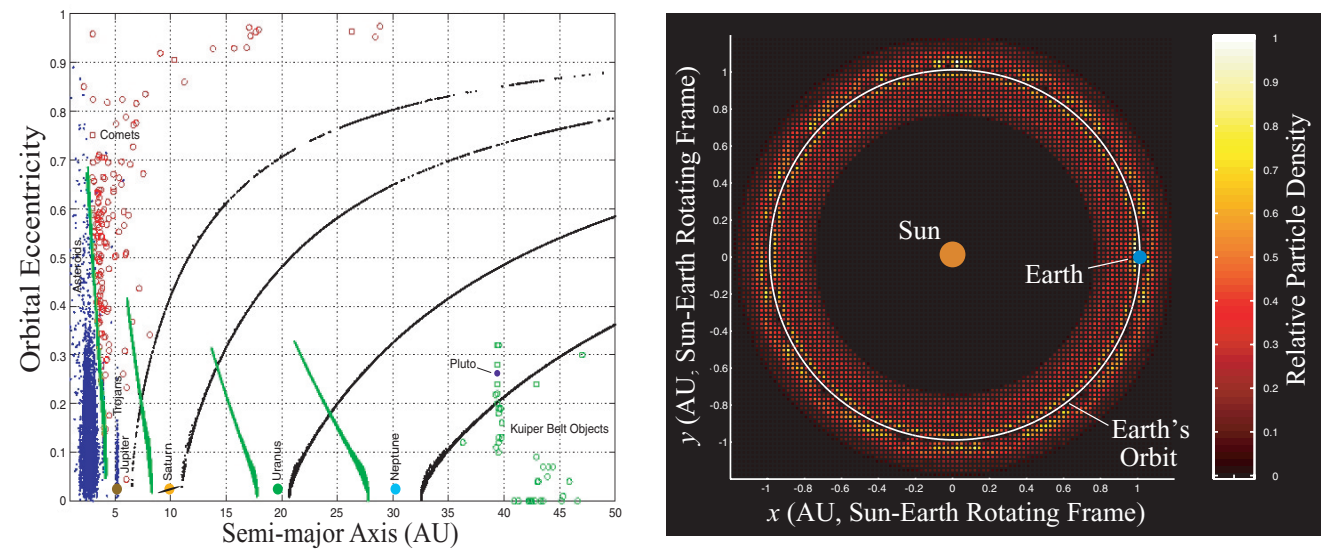

Figure 7.1: (a) Dynamical channels in the solar system. We plot the (local) semi-major axis versus the orbital eccentricity. We show the $L_{1}$ and $L_{2}$ manifolds for each of the giant outer planets. Notice the intersections between manifolds of adjacent planets, which leads to chaotic transport. Also shown are the asteroids, comets, and Kuiper Belt objects. (b) The zodiacal dust ring around the Earth's orbit, as modeled by Earth's $L_{1}$ and $L_{2}$ stable and unstable manifolds. We show the Sun-Earth rotating frame. Notice the "clumps" in Earth's orbit.

Potential Earth-impacting asteroids may utilize the dynamical channels as a pathway to Earth from nearby, seemingly harmless heliocentric orbits in resonance with the Earth. The same dynamics governing libration point space missions such as the Genesis Discovery Mission, which is on a natural Earth collision orbit, is also the dynamics that could bring unexpected Earth impactors. This phenomena has been observed recently in the impact of comet Shoemaker-Levy 9 with Jupiter, which was in 2:3 resonance with Jupiter (one of the resonances dynamically connected to the Jupiter region) just before impact.

Zodiacal Dust Cloud. Numerical simulations of the orbital evolution of asteroidal dust particles show that the Earth is embedded in a circumsolar ring of asteroidal dust known as the zodiacal dust cloud. Both simulations and observations reveal that the zodiacal dust cloud has structure. When viewed in the Sun-Earth rotating frame, there are several high density clumps ( $\sim 10 \%$ greater than the background) which are mostly evenly distributed throughout the Earth's orbit. The dust particles are believed to spiral in towards the Sun from the asteroid belt, becoming trapped temporarily in exterior mean motion resonances with the Earth. We suspect that the gross morphology of the ring is given by a simpler CR3BP model involving the homoclinic and heteroclinic structures (the dynamical channels) associated with $L_{1}$ and $L_{2}$ (Lo and Ross [1997]). See Figure 7.1(b).

The drag forces do not destroy the dynamical channel structure, but instead seem to lead to convergence onto the structure for particles spiraling in from the inner asteroid belt. Once trapped in a channel, the dynamics naturally lead to transport (via an Earth encounter) into the interior region, where drag forces dominate once more.

Acknowledgments. We thank Gerard Gómez and Josep Masdemont for helpful discussions and for sharing their wonderful software tools. We thank Donald Yeomans and Alan Chamberlin for the JPL Horizons integrator which generated the comet orbits. We thank Edward Belbruno and Brian Marsden for an advanced copy of their comet paper. We also wish to thank the following colleagues for helpful discussions and comments: Brian Barden, Julia Bell, Peter Goldreich, Kathleen Howell, Angel Jorba, Andrew Lange, Jaume Llibre, Regina Martínez, Richard McGehee, William McLaughlin, Linda Petzold, Nicole Rappaport, Ralph Roncoli, Carles Simó, Scott Tremaine, Stephen Wiggins, and Roby Wilson.

This work was carried out at the Jet Propulsion Laboratory and the California Institute of Technology under a contract with the National Aeronautics and Space Administration. The work was partially supported by the Caltech President's fund, the NASA Advanced Concepts Research Program, The Genesis Project, NSF grant KDI/ATM-9873133 and AFOSR Microsat contract F49620-99-1-0190. 


\section{References}

Abraham, R. and J.E. Marsden [1978] Foundations of Mechanics. Second Edition, Addison-Wesley.

Ascher, U.M. and L.R. Petzold [1998] Computer Methods for Ordinary Differential Equations and Differential-Algebraic Equations, SIAM.

Belbruno, E and B. Marsden [1997] Resonance hopping in comets, The Astronomical Journal 113(4), 1433-1444.

Belbruno, E and J. Miller [1993] Sun-perturbed earth-to-moon transfers with ballistic capture, Journal of Guidance, Control, and Dynamics 16, 770-775.

Bloch, A.M. and J.E. Marsden [1989] Controlling homoclinic orbits, Theoretical and Computational Fluid Mechanics 1, 179-190.

Bloch, A.M., N. Leonard and J.E. Marsden [1997] Stabilization of mechanical systems using controlled Lagrangians, Proc $C D C$ 36, 2356-2361.

Conley, C. [1963] On some new long periodic solutions of the plane restricted 3-body problem. Comm. Pure Appl. Math. 16, 449-467.

Conley, C. [1968] Low energy transit orbits in the restricted 3-body problem. SIAM J. Appl. Math. 16, 732-746.

Gómez, G., A. Jorba, J. Masdemont, and C. Simó [1991] Study Refinement of Semi-Analytical Halo Orbit Theory, Final Report, ESOC Contract No.: 8625/89/D/MD(SC), Barcelona, April, 1991.

Howell, C., B. Barden and M. Lo [1997] Application of dynamical systems theory to trajectory design for a libration point mission, The Journal of the Astronautical Sciences 45(2), April 1997, 161-178.

Kane, C, J.E. Marsden, and M. Ortiz [1999] Symplectic energy momentum integrators. J. Math. Phys., 40, 3353-3371.

Kane, C, J.E. Marsden, M. Ortiz and M. West [1999] Variational Integrators and the Newmark Algorithm for Conservative and Dissipative Mechanical Systems (Int. J. Num. Meth. Eng., to appear.).

Koon, W.S. and J.E. Marsden [1997] Optimal control for holonomic and nonholonomic mechanical systems with symmetry and Lagrangian reduction. SIAM J. Control and Optim. 35, 901-929.

Koon W.S., M.W. Lo, J.E. Marsden and S.D. Ross [1999a], Heteroclinic Connections between Liapunov Orbits and Resonance Transitions in Celestial Mechanics, Chaos, to appear.

Koon, W.S., M.W. Lo, J.E. Marsden and S.D. Ross [1999b], The Genesis Trajectory and Heteroclinic Connections, AAS/AIAA Astrodynamics Specialist Conference, Girdwood, Alaska, 1999, AAS99-451.

Koon, W.S., M.W. Lo, J.E. Marsden and S.D. Ross [2000], Shoot the Moon, AAS/AIAA Astrodynamics Specialist Conference, Florida, 2000, AAS 00-166.

Llibre, J., R. Martinez and C. Simó [1985] Transversality of the invariant manifolds associated to the Lyapunov family of perodic orbits near L2 in the restricted 3-body problem, Journal of Differential Equations 58, 104-156.

Lo, M. and S. Ross [1997] SURFing the Solar System: Invariant Manifolds and the Dynamics of the Solar System JPL IOM 312/97, 2-4.

Lo, M. and S. Ross [1998] Low energy interplanetary transfers using invariant manifolds of L1, L2 and halo orbits, AAS/AIAA Space Flight Mechanics Meeting, Monterey, California, 9-11 February 1998.

Lo, M., B.G. Williams, W.E. Bollman, D. Han, Y. Hahn, J.L. Bell, E.A. Hirst, R.A. Corwin, P.E. Hong, K.C. Howell, B. Barden, and R. Wilson [1998] Genesis Mission Design, Paper No. AIAA 98-4468.

Marsden, J.E. [1992], Lectures on Mechanics London Math. Soc. Lecture Note Series, 174, Cambridge University Press.

Marsden, J.E. and T.S. Ratiu [1999] Introduction to Mechanics and Symmetry. Texts in Applied Mathematics, 17, Springer-Verlag, 1994. Second Edition, 1999.

McGehee, R. P. [1969] Some homoclinic orbits for the restricted 3-body problem, Ph.D. thesis, University of Wisconsin.

Meyer, K.R. and R. Hall [1992] Hamiltonian Mechanics and the n-body Problem. Springer-Verlag, Applied Mathematical Sciences.

Moser, J. [1973] Stable and Random Motions in Dynamical Systems with Special Emphasis on Celestial Mechanics. Princeton University Press.

Szebehely, V. [1967] Theory of Orbits. Academic Press, New York/London.

Wiggins, S. [1990] Introduction to Applied Nonlinear Dynamical Systems and Chaos. Texts Appl. Math. Sci. 2, SpringerVerlag.

Serban, R. [1999] COOPT - Control and Optimization of Dynamic Systems - Users' Guide, Report UCSB-ME-99-1, UCSB Department of Mechanical and Environmental Engineering, 1999.

Serban, R., W.S. Koon, M.W. Lo, J.E. Marsden, L.R. Petzold, S.D. Ross and R.S. Wilson [2000] Optimal Control for Halo Orbit Missions, preprint. 